\title{
Knowledge flow analysis: the quantitative method for knowledge stickiness analysis in online course
}

\author{
Iveta Daugule ${ }^{1}$, Atis Kapenieks ${ }^{2}$ \\ ${ }^{1}$ Distance Education Study Centre, Riga Technical University, Riga, Latvia \\ ${ }^{2}$ Distance Education Study Centre, Riga Technical University, Riga, Latvia
}

\section{Article Info}

Received Dec $31^{\text {st }}, 2018$

\section{Keyword: \\ e-Learning \\ Knowledge flow analysis \\ Knowledge properties \\ Knowledge stickiness \\ Online courses \\ Student motivation}

\begin{abstract}
The aim of this study is to better understand the feasibility of using technological solutions to detect specific knowledge descriptors in online learning systems. Understanding the relevance of specific knowledge in the context of online courses is important for the architecture of the content of the course and for improving the effectiveness of the learning process. By identifying the specific nature of the knowledge flow in a timely manner, it is possible to better adapt the course content to the needs of the student and to ensure that the time spent on learning is used effectively. In the case of this tailored course content, it is expected that in a given course, it would be possible to learn more content than a time-like course where knowledge stickiness has not been taken into account. By using calculations and excluding the possibility of a subjective view as far as possible, authors are clarifying the nature of knowledge for each of the competencies. Automated evaluation of knowledge properties would significantly facilitate the learning process by allowing better management of the knowledge flow. Improving the effectiveness of preparing training materials would be a significant benefit from the development of such a solution.
\end{abstract}

\section{Corresponding Author:}

Iveta Daugule,

Distance Education Study Centre

Riga Technical University

Kronvalda bulvāris 1, Riga, LV-1010

Email: iveta.daugule_1@ rtu.lv

\section{Introduction}

The aim of this study is to better understand the feasibility of using technological solutions to detect specific knowledge descriptors in online learning systems. Automated evaluation of knowledge properties would significantly facilitate the learning process by allowing better management of the knowledge flow. Improving the effectiveness of preparing training materials would be a significant benefit from the development of such a solution.

In the authors' view, automated identification of the knowledge parameters would bring considerable benefits in the following situations:

- optimizing the amount of work necessary for the development and development of the content of the training course. After identifying non-sticky knowledge, the relevant training materials may be recognised as useful and their efforts may be devoted to the development of training materials that identified by the system as containing sticky knowledge;

- optimize the consulting work with students, allowing the teacher to see which questions to prepare more thoroughly; 
- the above applies also to the conduct of the training process itself, giving the teacher instructions beforehand, which fields of knowledge need to be prepared in depth, and which, in the form of interactive training, can allow students to learn by themselves, in collaboration with each other and in addition to the direct task, also by strengthening their communication and other skills that are relevant to the study process.

During this study, a more extensive research was conducted on properties that describe knowledge stickiness. The aim of this research was to comprehend possible technological solutions to detect specific knowledge descriptors in online learning systems.

The study was conducted at the Basic Business study course. During this course, the student learns 7 competencies - actuality, technology, marketing, competition, finances, risks, and the ability to implement a business idea.

One of the course tasks was participation in the peer-review process. During the peer review process, the student reviewed 3 of his fellow study members' works and provided recommendations for their improvement. After performing this task, he or she gained the opportunity to familiarize himself / herself with the recommendations of his / her fellow study members for improving his /her own work. Then the student received the next task: to improve his / her work, taking into account the recommendations of the study members and to upload his / her now improved work into the system. The knowledge stickiness was determined by recording the student's initial knowledge and the student's final knowledge, after the conclusion of the peer review process between students. In order to carry out an assessment of the knowledge stickiness of each different competency, the student's uploaded essay was assessed separately for each of the 7 competencies, for both the initial and final versions.

By using calculations and excluding the possibility of a subjective view as far as possible, authors are clarifying the nature of knowledge for each of the competencies. Automated evaluation of knowledge properties would significantly facilitate the learning process by allowing better management of the knowledge flow.

\section{The Scope of Study}

Evaluating the use of the term "stickiness" in the context of online learning content, the authors found that "stickiness" as a descriptor is used for two types of situations.

Theobald points out that the "stickiness" meaning of attractiveness came about very soon after the first websites were created - their authors noticed that the content had to meet the expectations of the reader to keep his attention [1]. When it comes to online content, stickiness is used as a trait of mutual attraction - to what extent the content is binding on the reader to the extent that the student is intrigued by what he is learning. In this context, it is a descriptor of how long a student wants to spend time getting acquainted with the content of the proposed training and how he wants to return to it. In this context, the desired situation is described as a "Velcro type" situation where a bi-directional relationship is formed between the student and the learning environment [2].

This understanding is more widely used in the context of online content, realizing that good content makes the reader spend as much time as possible reading it, and has a positive impact on the learning outcomes achieved.

Although from the authors' point of view, the second meaning of the term "stickiness" in the context of organizing the knowledge flow is also important in evaluating the content of online learning. This meaning is more often used in the context of organizational knowledge transfer and its management.

By Mooradian [3], tacit knowledge has a specific role to play in knowledge management: it is a factor in knowledge transfers that explains or predicts the stickiness of the transfer. Explicit knowledge in organizational context is treated as a kind of surface pool that is easier to detect and capture, but it represents only a fraction of the organizational knowledge. At the same time tacit knowledge is seen as the challenge to organizations that want to spread knowledge throughout the organization or spur greater innovation and is treated as a reserve deposited deep within the ground that needs to be detected and then pumped out [3].

The authors believe that it is important to be aware in this context that the same type of knowledge can be not only within an organization but also in an online course. In addition, if this type of knowledge is not identified in a timely manner, the student may lack the opportunity to acquire it at the required knowledge level, which may result in failure to achieve the set learning goals. 
According to Nissen, tacit knowledge is associated with stickiness, and is difficult to imitate, distribute and slow to move [4]. Szulanski [5] points out that the adjective "sticky" is used as a synonym for immobility, inertness, and inimitability, and with meaning "gluey". He points out that in the context of knowledge transfer, "sticky transfers" refers to situations where vigilance and effort are required to detect and overcome difficulty. Knowledge that transmits with much more effort than others is perceived as sticky. Also stickiness is an attribute of knowledge transfer, which reflects both the characteristics of the transfer situation and knowledge that being transferred [5].

In the authors' view, the stickiness in the context of knowledge transfer is worth exploring more broadly, by using this context for the knowledge that is transferred through online courses. Understanding the relevance of specific knowledge in the context of online courses is important for the architecture of the content of the course and for improving the effectiveness of the learning process. By identifying the specific nature of the knowledge flow in a timely manner, it is possible to better adapt the course content to the needs of the student and to ensure that the time spent on learning is used effectively. In the case of this tailored course content, it is expected that in a given course, it would be possible to learn more content than in a course of equal length where knowledge stickiness has not been taken into account.

\section{Methodology}

During this study, a more extensive research was conducted on properties that describe knowledge stickiness in the context of knowledge transfer, by using this context for the knowledge that is transferred through online courses. The aim of this research was to comprehend possible technological solutions to detect specific knowledge descriptors in online learning systems.

The study was conducted at the Basic Business study course. During this course, the student learns 7 competencies - actuality, technology, marketing, competition, finances, risks, and the ability to implement business idea.

This study course is based on the Open edX platform and the peer-review mechanism available within it. The mechanism applied ensures that each of the students provides recommendations to the three randomly selected members of their studies and then receives the advice of other students in the same way.

In order to determine the characteristics of the knowledge flow, a situation is created in which it is possible to assess the student's initial knowledge, then a mechanism is created that allows supplementing this knowledge. At the end of the assignment, the student's level of knowledge is recorded. It was provided with the following steps:

- Step 1. The student, using existing knowledge, prepares an essay describing his / her business idea. Characteristics and solutions to seven problems are included in the idea description. These positions correspond to the 7 competencies acquired during the course. At this point, the student's knowledge is fixed.

- Step 2. The student uploads his / her essay into the online system, where it is included in the peerreview process. It undergoes the peer-review process and recommendations are provided by 3 study members, whereas the student gets acquainted with the works of 3 of his / her fellow students. Upon completion of the mutual review of the work, the student will receive the recommendations given to him / her. During the peer-review, students maintain mutual anonymity.

- Step 3. The student re-evaluates his / her work and implements the recommendation at his/her own discretion. The work is then re-uploaded to the system. At this point, the student's final knowledge is recorded.

For the process representation, see Figure 1. 


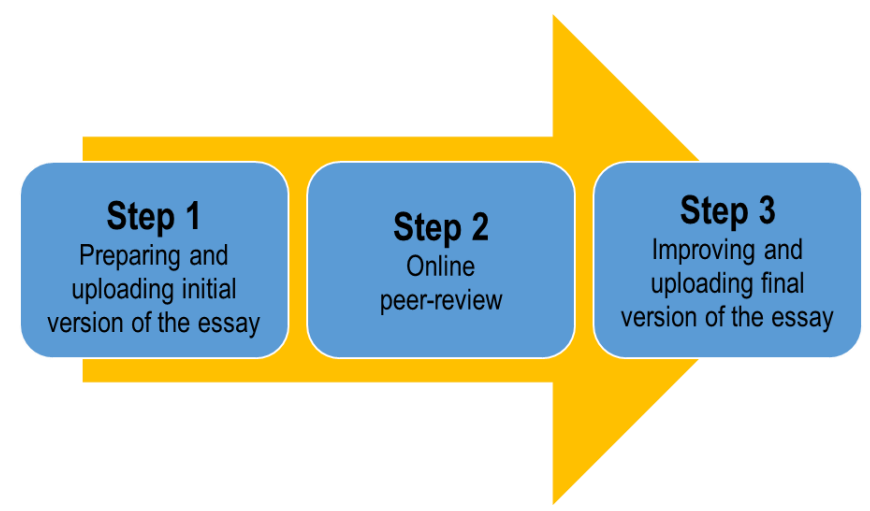

Figure 1. Peer-review process.

The authors analysed the texts submitted by students in order to determine the changes made during the learning process by the character count and word count, concerning each individual competence in both the initial and final versions. Calculations were then made to determine the differences between the number of characters and the number of words in the initial and final versions of the essays.

The mean value of this difference and the standard deviation of these values were then calculated separately for each competency.

The same set of data used in authors' previous study was used in this study, as well [6]. In the abovementioned study [6], the same set of essays' initial and final versions were evaluated by comparing them to a similar principle. The initial and final versions of the essays were compared by making a separate assessment of each competence, calculating the progress made, its mean value and determining the standard deviation of that value. Unlike the methodology of this study, the evaluation was carried out in a qualitative way - the instructor got acquainted with the content of the essays submitted by the students and rated each competence with a grade. Subsequently, calculations for determining the knowledge stickiness were also made from the differences of these grades.

The authors cross-validated the results of both studies. Accordingly, to this comparison of results authors assessed deficiencies of each method, to find reasonable solutions for their further development. Against this background, the authors also drew attention to the factors that had potentially affected the results of the previous study [6].

\section{Results}

The research was conducted on properties that describe knowledge stickiness in the context of knowledge transfer, by using this context for the knowledge that is transferred through online courses. In the study, the authors concluded that different knowledge in the course has different stickiness, which can be identified. This stickiness is characterized by the level of effort required to succeed in the learning.

In their previous study [6], the authors' assessed the knowledge stickiness in a subjective way - each of the essay sections corresponding to each competence was rated by the course instructor with a grade. The evaluation was made for both initial and final versions. After that the mean value and the standard deviation of the grades was determined and the progress made by the students was calculated. This study [6] found out that with the mean value of gained progress as 0.39 and the standard deviation progress as -0.01 the stickiest knowledge in this online course is the "Technology". The most explicit knowledge, judging by the progress in the mean value as 1.1 , is found the skill "Competition".

During this study, the authors carried out quantitative analysis of the essays submitted by students, adding up and comparing the number of characters and words in the both initial and final versions of the essays submitted by students. Characters and words were counted and analysed for each competence separately. In the next step, the difference between these parameters for each of the competencies for the original and final version and its mean value was calculated.

Evaluating the obtained results, the authors concluded that there was no evidence found of a general direct correlation between the progress of the evaluation of the submitted works within the framework of the study and the changes made by the students to the text of the essays during the review. However, the results clearly allowed us to identify knowledge with distinct stickiness characteristics, as evidenced by both the subjective assessment carried out previously [6] and the quantitative analysis carried out in this study. 
Same as the subjective assessment [6], the quantitative assessment highlighted two of the seven competences as distinct from the common knowledge flow. One of these competences is Technology. It is characterized by very small changes compared to average grade progress $(0.39)$ and standard deviation parameter changes (0.01) [6], as well as pronounced effort level parameters: 19.82 words per grade or 145.35 characters per grade (see Figure 2, the trend line).

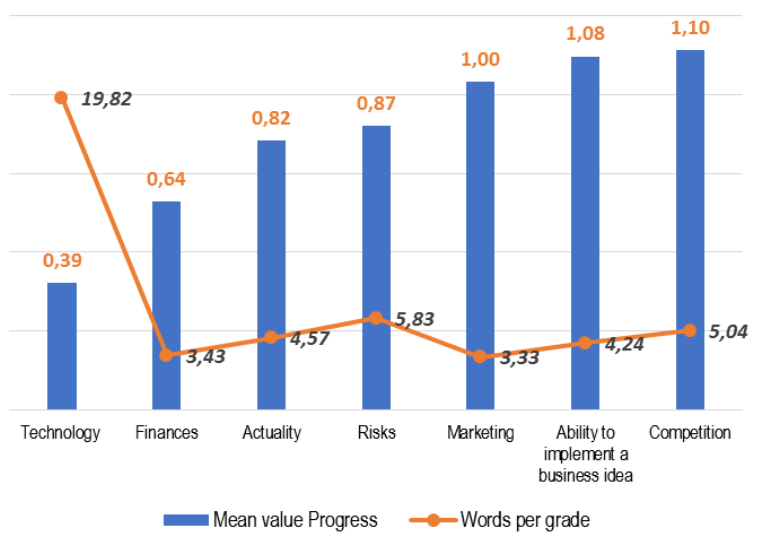

Figure 2. Comparison between "Grade mean value progress" [6] and calculated effort level "Words per grade".

The second competence that emerged significantly on the trend line is Risks. Although, according to previous data [6], the average grade value progress is medium (0.87), it is characterized by a marked change in the standard deviation parameter $(-0.74)$, which already highlighted this competence among others in the previous study [6]. Unlike others, following the peer-review essay and the recommendations it received, there was a significant reduction in the grade range in a positive direction [6].

In turn, in this study, competence Risk describes the second largest level of effort to be applied to acquire this skill. Effort level in this case is the number of characters to be written to improve on a scale of one ball: 5.83 words per grade or 42.84 characters per grade (see Figure 3, the trend line).

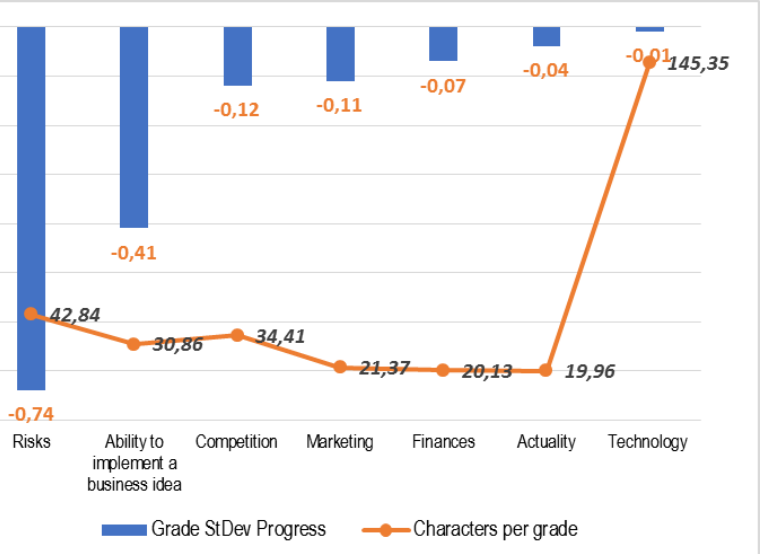

Figure 3. Comparison between "Grade standard deviation value progress" [6] and calculated effort level "Characters per grade".

When evaluating the properties of other competencies, no clear and unambiguous features of knowledge stickiness were found. At the same time, when analyzing the aggregated data, several factors were found that could have influenced the results obtained.

The analyzed works were presented in the form of essays; thus, the works did not have a uniform structure. This situation also had an impact on the stickiness analytics results.

This factor could have influenced the qualitative assessment with a grade. When evaluating the essays, the situation was possible where a positive evaluation on the basis of one text fragment was assigned to more than one competence. The same factor could also have influenced the quantitative assessment - in this case, one text fragment was explicitly attributed to only one - the closest competence. 
In a qualitative assessment, one sentence could be attributed to more than one competence and considered to improve both. In the quantitative assessment, the same sentence was strictly linked to one of the most relevant competences.

The situation described above affected the results. Accordingly, only those situations where competence characteristics were significantly different from others clearly emerged. In other cases where the descriptors are closer together, these indicators are unclear.

Also, by re-analyzing the content of student works, the authors found that in some cases the instructor, in qualitative assessment, had placed a higher grade on the final version for the specific competence, although the quantitative evaluation showed that the text had not changed and the only improvement was the document formatting correction, making it more visually appealing.

According to the above, in the authors' view, it would be possible to obtain more precise characteristics if the comparative data were submitted in line with a defined pattern, where the boundaries of different competencies would be strictly separated.

In order to do this, it is necessary to improve the technical solution of the respective online course by providing another form of submission. At the same time, it is necessary to clarify the task given to the student by replacing the free-form essay format with a report that is structured in chapters. Performing these activities would be more convenient for the student and at the same time it would be a more suitable technical solution for the knowledge stickiness analysis.

By comparing the methods applied in this study, the authors performed a correlation analysis between the results obtained by "Words per grade" and "Characters per grade". The calculated correlation is 0.99 , so it is considered that the results are directly and strongly correlated. Based on this result, the authors believe that it is sufficient to count and include only one of these factors in further analysis.

\section{Results}

In the study, the authors concluded that different knowledge in the course has different stickiness, which can be identified. This stickiness is characterized by the level of effort required to succeed in the learning.

In the authors' view, stickiness in the context of knowledge transfer is worth exploring more extensively, by using this context for the knowledge that is transferred through online courses. Understanding the relevance of specific knowledge in the context of online courses is important for the architecture of the content of the course and for improving the effectiveness of the learning process.

By identifying the specific nature of the knowledge flow in a timely manner, it is possible to better adapt the course content to the needs of the student and to ensure that the time spent on learning is used effectively. In the case of this tailored course content, it is expected that in a given course, it would be possible to learn more content than in a course of equal length where knowledge stickiness has not been taken into account.

The calculated correlation between "Words per grade" and "Characters per grade" is 0.99 , so it is considered that the results are directly and strongly correlated. Based on this result, it is sufficient to count and include only one of these factors in further analysis.

In the authors' view, it would be possible to obtain more precise characteristics if the comparative data were submitted according to a defined pattern, where the boundaries of different competencies would be more strictly separated.

It is necessary to clarify the task given to the student by replacing the free-form essay format with a report that is structured into chapters. Performing these activities would be more convenient for the student with and in the same time it would be a more suitable technical solution for the knowledge stickiness analysis.

\section{Acknowledgements}

This research has been supported by a grant from the European Regional Development Fund (ERFD/ERAF) project "Technology Enhanced Learning E-ecosystem with Stochastic Interdependences - TELECI", Project No.1.1.1.1./16/A/154.

\section{References}

[1] Theobald, T. On Message: Precision Communication for the Digital Age. Kogan Page, Limited, 2013.

[2] Robinson A., Cook D., "Stickiness": gauging students' attention to online learning activities. Information and Learning Science, Vol. 119, Issue: 7/8 pp.460-468, https://doi.org/10.1108/ILS-032018-0014 
[3] Mooradian, N. (2005) "Tacit knowledge: philosophic roots and role in KM", Journal of Knowledge Management, Vol. 9 Issue: 6, pp.104-113, https://doi.org/10.1108/13673270510629990

[4] M. E. Nissen, "Harnessing Knowledge Dynamics: Principled Organizational Knowing \& Learning" IRM Press, 2005

[5] Szulanski G., Sticky Knowledge: Barriers to Knowing in the Firm. SAGA Publications. 2002

[6] Daugule, I., Kapenieks, A., Collaborative Knowledge Flow - Mapping the E-Learning Environment, EDULEARN17 Proceedings (2017), pp. 3304-3311. 